Check for updates

The BMJ

Cite this as: $B M J 2020 ; 371: m 3846$ http://dx.doi.org/10.1136/bmj.m3846 Published: 01 October 2020

\section{Covid-19: Vaccine roll out could take a year and will require difficult prioritisation decisions}

\author{
Elisabeth Mahase
}

Even after a covid-19 vaccine is found to be effective, it could still take a year to reach the general public, according to experts.

And the roll out of any vaccine will be accompanied by difficult decisions about who should be prioritised to receive it, warned the team from the Royal Society DELVE Initiative in a report outlining the key considerations for developing, evaluating, manufacturing, and distributing a vaccine against SAR-CoV-2.

"Even when the vaccine is available, it does not mean within a month everybody will be vaccinated. We're talking about six to nine months to a year after a vaccine is approved," said Nilay Shah, head of chemical engineering at Imperial College London, speaking at a Science Media Centre briefing on 1 October.

Shah explained that if you divide the UK population into five year age groups and then those over 80 , and you can vaccinate a group within two weeks, it would take about eight months to vaccinate all groups, and it could take longer. That would also depend on having stock built up and ready to distribute and trained staff available to focus solely on vaccinations.

Rolling out a vaccine over a year will mean that decisions must also be made about which groups get vaccinated before others. Charles Bangham, chair of immunology and co-director at the Institute of Infection at Imperial College London, said, "There will have to be some prioritisation, that's going to be a difficult choice that will have to be made by policy makers. How do they distribute it to the best advantage and to which high risk groups first? Elderly people in care homes, healthcare workers, and so on.”

Bangham added that people must also realise that an effective vaccine will not mean the virus goes away. "It's not going to end the infection completely. We hope that it will be possible to bring it under control, as we do to an extent with seasonal influenza," he said.

The group has called for criteria for vaccine prioritisation to be "defined and made explicit." There should then be "public dialogue and engagement to manage expectations and understanding of vaccine effectiveness, safety, side effects, availability, and access.”

\section{Ensuring uptake}

Aside from the difficulties of manufacturing, storing, and distributing a vaccine, ${ }^{2}$ success of an immunisation programme will rely heavily on public trust and access. As such, it is important that vaccine hesitancy and barriers to uptake are tackled, the group said.

While praising the speed at which vaccine candidates - of which there are over 200-have been developed, the report warned that "strict regulation of clinical trials and robust licensing rules must be maintained in spite of the urgency and requirement for speedy implementation."

It added, "The history of vaccine development is littered with candidates that have failed at this late stage. If vaccines were deployed outside clinical trials before safety and efficacy have been fully established and prove to be ineffective or cause rare but severe side effects during the larger scale roll out, they could cause substantial harm and damage public confidence in other vaccines.”

The authors have recommended "clear, transparent communication" to tackle rational doubts and to enable informed decision making. They said such communication "should not hide the potential limitations of vaccines" as although negative or complicating factors could lower uptake, "their discovery post-rollout is likely to have a far greater negative impact on uptake.”

The team also called for a population-wide strategy that tackles the social and economic disincentives to vaccination, such as the need to take time off work or secure childcare to get a vaccine, as these barriers are "most likely to be greatest for those in society most vulnerable to covid-19." They recommended local distribution of the vaccine through community workers as well as healthcare workers and with evening and weekend opening hours.

Charging for vaccination or withholding vaccines based on citizenship are not recommended, but any strategy should involve community leaders to maximise uptake "by tackling religious, cultural, or other context specific concerns."

The DELVE Initiative. (2020), SARS-CoV-2 vaccine development \& implementation; scenarios, options, key decisions. DELVE Report No. 6. 1 October 2020. https://rs-delve.github.io/reports/2020/10/01/covid19vaccination-report.html.

2 Mahase E. Covid-19: What do we know so far about a vaccine?BMJ 2020;369:m1679. doi: 10.1136/bmj.m1679 pmid: 32340998 